\title{
Barrier-protective function of intestinal epithelial Toll-like receptor 2
}

\section{E Cario $^{1}$}

The intestinal epithelial cell (IEC) barrier plays an important role in maintaining mucosal immune homeostasis. Dysregulated IEC barrier function appears to trigger and perpetuate inflammation in inflammatory bowel diseases (IBD). Novel risk variants in the Toll-like receptor 2 (TLR2) gene have previously been associated with a more severe disease phenotype in a subgroup of IBD patients. Recent studies have provided important insights of the commensal and host defense mechanisms to maintain functional barrier integrity of the intestinal epithelium through TLR2. Deficient TLR2 signaling may imbalance commensal-dependent intestinal epithelial barrier defense, facilitating mucosal injury and leading to increased susceptibility of colitis. Treatment with a synthetic TLR2 ligand significantly suppresses mucosal inflammation by efficiently protecting tight junction-associated integrity of the intestinal epithelium in vivo. These beneficial effects may be supplemented by TLR2-induced anti-inflammatory immune responses (such as interleukin-10 production) in lamina propria mononuclear cells. Thus, cell-specific TLR2 targeting may offer a novel therapeutic approach to human IBD therapy by protecting IEC barrier function.

\section{INTRODUCTION}

The epithelial lining of the intestine constitutes an anatomic as well as immunologic barrier that forms a bipolar interface between the diverse populations of luminal microbes and immune cells of the underlying lamina propria. The intestinal epithelial cell (IEC) barrier must exert highly complex and dynamic functions of precise selection of luminal contentsexcluding potential host-threatening agents while allowing hostbeneficial substances (e.g., nutrients) to permeate. Importantly, it must maintain hyporesponsiveness to harmless commmensals, but rapidly initiate controlled immune responses once luminal pathogens are specifically recognized. Thus, this delicate barrier acts as a crucial gatekeeper toward the "outside world" as well as essential coordinator of immediate host defenses to maintain mucosal immune homeostasis.

IEC maintains close contact with each other through the formation of tight junctions (TJs). Increased epithelial permeability due to $\mathrm{TJ}$ alterations may initiate and perpetuate intestinal inflammation in human inflammatory bowel diseases (IBDs) ${ }^{1,2}$ as well as some murine models of colitis that resemble, in certain aspects, human IBD (including dextran sulfate sodium (DSS) colitis). ${ }^{3}$ By crossing such leaky IEC barriers, luminal antigens can gain access to the subjacent lamina propria that result in chronic activation of $\mathrm{T}$ cells and disturbance of the natural tolerance mediated by regulatory $\mathrm{T}$ cells and immunosuppressive cytokines. ${ }^{4}$ In contrast, TJ preservation and reassembly may significantly enhance IEC barrier function, thus essentially contributing to protection of the underlying lamina propria from invasion of detrimental antigens. For instance, glucocorticoids, which are successful in human IBD therapy, have been shown to modulate TJs associated with induction of retightening and restitution of the intestinal TJ barrier defect in Crohn's disease patients through their direct stimulatory effects on MLCK. ${ }^{5}$ Emerging evidence exists that commensals directly assist the host to strengthen IEC barrier function thus efficiently inhibiting bacterial invasion and consequently attenuation of intestinal inflammation in IBD. ${ }^{6-8}$ To further understand the precise innate immune mechanisms of such commensal-mediated IECprotective key features that critically maintain TJ-associated barrier homeostasis, our group has recently pursued a sequence of experimental studies, ${ }^{9-12}$ which is briefly summarized and discussed here within the context of the current literature.

\section{CELL-SPECIFICTLR2 SIGNALING INTHE INTESTINAL MUCOSA}

Toll-like receptors (TLRs) comprise a class of transmembrane pattern-recognition receptors that play a key role in microbial recognition, induction of antimicrobial genes, and control of adaptive immune responses. ${ }^{13}$ TLRs are type I transmembrane

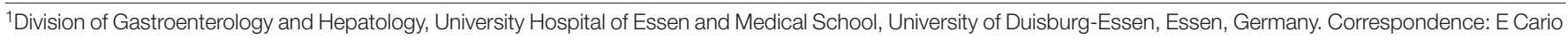
(elke.cario@uni-duisburg-essen.de) 


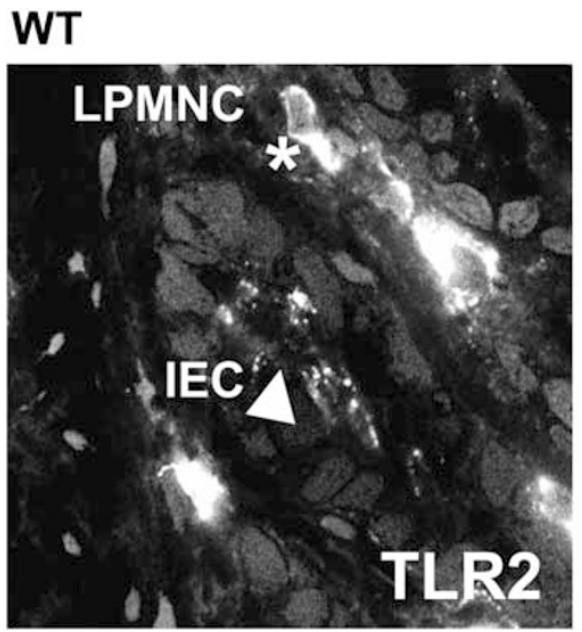

\section{TLR2-I-}

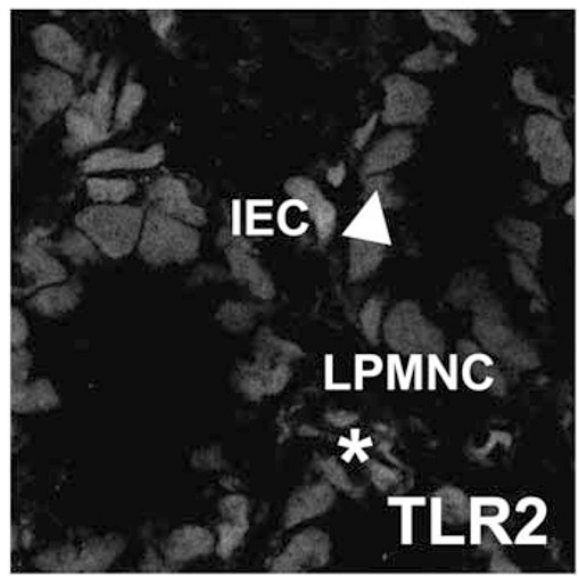

Figure 1 Toll-like receptor 2 (TLR2) protein expression in intestinal epithelial cells (IEC) and lamina propria mononuclear cells (LPMNC) of healthy mouse gastrointestinal mucosa. TLR2 (fluorescein isothyocyanate; FITC) is constitutively expressed in IEC (arrow) or LPMNC (star) in colons of healthy wild-type (WT) but not TLR2-deficient mice, as assessed by confocal immunofluorescence analysis of methanol/acetone-fixed frozen sections (×63/1.4, oil, scan zoom 1.0). Nuclei were counterstained with propidium iodide (rhodamine).

glycoproteins with a single transmembrane region and a conserved intracellular domain. ${ }^{14}$ TLR2, one member of the TLR family, recognizes conserved molecular patterns associated with both Gram-negative and Gram-positive bacteria, including lipopeptides/lipoproteins, lipoteichoic acid, zymosan, and components of peptidoglycan. Triacylated lipopeptides are recognized by TLR2/TLR1 -, whereas diacylated lipopeptides use TLR2/TLR6 heteromers for signaling. The synthetic triacylated lipohexapeptide analog $N$-palmitoyl-S-[2,3-bis(palmitoyloxy)(2RS)-propyl]-(R)-cysteinyl-seryl-(lysyl)3-lysine ${ }^{15}$ (Pam ${ }_{3}$ CysSK4, named hereafter PCSK) represents an example for a specific TLR2 ligand, which in part signals through TLR1, but independently of TLR6. ${ }^{16}$ Binding of PCSK to TLR2 occurs in a lipid-binding pocket that is formed at the convex face of the junction between the central and C-terminal LRR domains and that accommodates the two ester-bound lipid chains of PCSK. ${ }^{17}$ The third amide-bound lipid chain of PCSK may be inserted into a hydrophobic channel in TLR1. PCSK-induced formation of the TLR1-TLR2 heterodimer facilitates TIR domain activation and subsequent downstream signaling. Interaction with non-TLR receptors may further diversify the ligand recognition potential of TLR2, including dectin- $1^{18}$ or CD36. ${ }^{19}$

TLR2 is differentially expressed by many distinct cell types throughout the healthy gastrointestinal tract, including IEC $^{9}$ and certain subsets of lamina propria mononuclear cells (LMPNC; Figure 1). Novel risk variants in the TLR1, TLR2, and TLR6 genes have been associated with a more severe disease phenotype in IBD patients of a selective Belgian population, ${ }^{20}$ yet their functional impact on disease pathogenesis remains unresolved. There is emerging evidence that cell-type-specific differences in modulation, signaling and functional effects of TLR2 within the intestinal mucosa exist (Figure 2). Upon ligand recognition, tyrosine phosphorylation of TLR2 leads to assembly of a multiprotein complex that includes Rac1 and phosphatidylinositol 3-kinase (PI3K) in primary immune cells ${ }^{21}$ in the lamina propria. Downstream cascades involve recruitment of the cytoplasmic adaptor proteins Mal and MyD88 to the TLR2 complex, ${ }^{22}$ which results in activation of nuclear factor- $\kappa \mathrm{B}(\mathrm{NF}-\kappa \mathrm{B})$ and subsequent production of many cytokines and chemokines. TLR2-mediated induction of NFKB may be further enhanced by the scaffold protein AKAP13. ${ }^{23}$ TLR2 is a potent inducer of anti-inflammatory interleukin-10 (IL-10), which critically inhibits multiple macrophage and dendritic cell (DC) effector functions, thus limiting exaggerated immune responses. IL10 also abolishes chronic inflammation-related endoplasmic stress response mechanisms by modulating ATF- 6 transcriptional activity. ${ }^{24}$ Importantly, TLR2-induced IL-10 secretion may modulate cell signaling through other TLRs. For instance, enhanced IL-10 production by TLR2-primed DCs abolishes TLR3/4-mediated production of proinflammatory IP-10 and IL-12p $35,{ }^{25}$ but further amplifies IL-10 production upon TLR4 restimulation. ${ }^{26}$ In addition, TLR2 also activates regulatory $\mathrm{T}$ cells via PI3K to secrete transforming growth factor- $\beta,{ }^{27}$ which is essential for tissue healing and epithelial restitution in the intestine. In contrast, in isolated mouse splenocytes, TLR2 stimulation has been shown to directly induce $\mathrm{T}_{\mathrm{H}} 1$-mediated immune responses, ${ }^{28}$ but the effects of local engagement of TLR 2 on effector T cells in the lamina propria remain to be delineated in detail. TLR2 signaling may be negatively influenced by alterations of coexistent patternrecognition receptors. Presence of a CARD15 mutation - which has been shown to be associated with increased Crohn's disease susceptibility-imbalances TLR2-mediated NFKB activation in antigenpresenting cells, thus leading to mucosal inflammation through exaggerated interferon- $\gamma$, IL-12, and IL-23 production, ${ }^{29}$ which in turn may further suppress mucosal TLR2-mediated anti-inflammatory IL-10 production via paracrine loops. ${ }^{30}$

\section{TLR2-MEDIATED IEC BARRIER INTEGRITY}

In contrast to lamina propria mononuclear cells (LPMNC), the intestinal epithelium is constantly exposed to omnipresent TLR2 ligands. Potential proinflammatory responses via TLR2$\mathrm{NF \kappa B}$ are instantly suppressed by diverse mechanisms, such as 


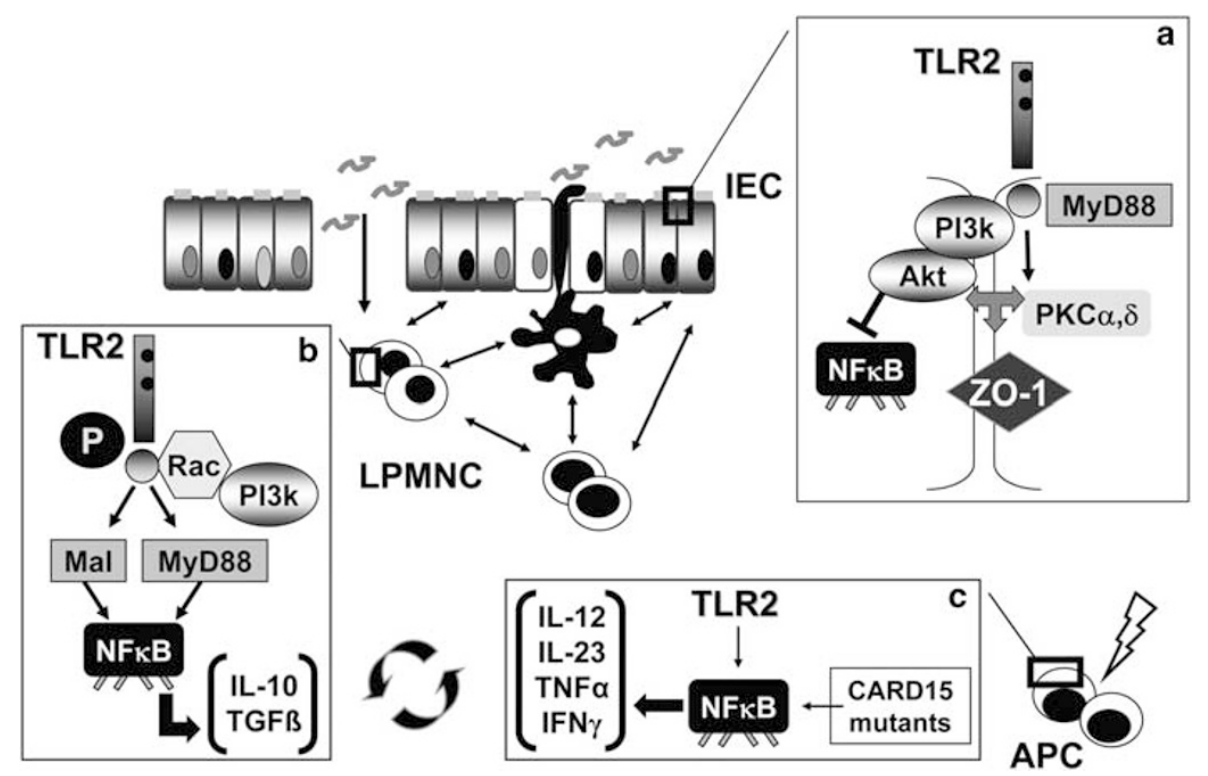

Figure 2 Current concept of physiological effects of mucosal barrier-protective Toll-like receptor 2 (TLR2) in the healthy gastrointestinal tract. To maintain commensal and mucosal homeostasis, mucosal TLR2 exerts several cell-type-specific features of barrier protection. Intestinal epithelial TLR2 critically controls mucosal inflammation by directly preserving tight junction (TJ)-associated barrier integrity at the frontline of host defense. In addition, dendritic cell TLR2 engagement promotes antigen capture and processing. TLR2 also modulates regulatory T cells by controlling adaptive immune responses. (a) In intestinal epithelial cells, TLR2 stimulation efficiently preserves zonula occludens-1 (ZO-1)-associated barrier integrity against stress-induced damage, which is controlled by positive signaling crosstalk between PI3K-Akt and conventional protein kinase $\mathrm{C}$ (PKC) isoforms via MyD88. In parallel, the PI3K/Akt pathway limits proinflammatory TLR2-signaling through the Mapk-NFKB pathway. (b) In lamina propria mononuclear cells (LPMNC), tyrosine-phosphorylated TLR2-TLR1 or TLR2-TLR6 receptor complexes activate signaling events via Rac1 and phosphatidylinositol 3-kinase (PI3K). Downstream recruitment of the adaptor proteins Mal or MyD88 lead to diverse immune responses through cytokine production (such as anti-inflammatory interleukin-10 (IL-10)) via transcriptional activation of nuclear factor- $\mathrm{KB}(\mathrm{NF} \kappa \mathrm{B})$. (c) In antigen-presenting cells, TLR2-NF $\mathrm{KB}$ signaling may be altered by CARD15 mutations, leading to exaggerated immune responses through proinflammatory cytokine production (such as IL-12 or interferon- $\gamma($ IFN $\gamma))$. Further studies are needed to dissect in detail the complex cell-cell interactions and signaling effects via TLR2 within the intestinal mucosal barrier in health versus disease. IEC, intestinal epithelial cell; TGF $\beta$, transforming growth factor- $\beta$; TNF $\alpha$, tumor-necrosis factor- $\alpha$.

high levels of Tollip, activation of the PI3K/Akt pathway, and A20. ${ }^{8,10,12,31,32}$ In a series of studies, we have recently demonstrated that intestinal epithelial TLR2 signals, through different pathways, eliciting immune responses which ideally reflect the functional necessities at this strategic location of frontline host defense to primarily protect barrier function (Figure 2).

Initially, we have observed that TLR2 stimulation selectively enhances transepithelial resistance (an essential measure of barrier integrity) of IEC monolayers in vitro, which was prevented by pretreatment with protein kinase $\mathrm{C}(\mathrm{PKC})$-selective antagonists. ${ }^{11}$ This effect correlated with $P K C \alpha / \delta$-mediated apical tightening and sealing of zonula occludens-1 (ZO-1), which serves as a major scaffolding $\mathrm{TJ}$ protein, cross-linking claudins, occludins, and the E-cadherin/catenin complex with the actinomyosin ring of the epithelial cell. ${ }^{33,34}$ Both PKC isoforms are known to specifically interact with PI3K/Akt activity. ${ }^{35}$ Indeed, in parallel to PKC phosphorylation, the Akt $\rightarrow$ p70S6K $\rightarrow$ ribosomal protein S6-pathway was significantly induced in response to selective TLR2 ligands. Using an ex vivo 3D-culture model of primary murine IEC on Matrigel, we functionally demonstrated that TLR2 stimulation efficiently preserves ZO-1-associated barrier integrity of IEC against stress-induced damage, which was critically controlled by the PI3K/Akt-pathway via MyD88. ${ }^{12}$

Entire loss of TLR2 has previously been shown to lead to dramatic exacerbation of intestinal inflammation in DSS colitis with high morbidity and mortality, ${ }^{12,36}$ suggesting that TLR2 signaling may exert important, yet to be identified, immune-protective responses in the intestinal mucosa. Consequently, we demonstrated ${ }^{12}$ that commensal-mediated TJ protection of the intestinal epithelial barrier is primarily impaired in the absence of TLR2 in DSS colitis. Inflammatory stress in mice, deficient of TLR2, induced early and complete TJ-loss of the intestinal epithelial barrier, which was not evident in wild-type mice. ZO-1 disassembly was associated with marked disruption of E-cadherin from cell-cell adhesion contacts. Deficient PI3K/Akt signaling and loss of junctional anchorage in the absence of TLR2 resulted in subsequent apoptotic shedding of IEC, thus increasing barrier permeability and aggravating mucosal inflammation. These findings have recently been confirmed in another murine model of intestinal epithelial barrier injury. ${ }^{37}$ TLR2deficient mice, infected with the bacterial pathogen Citrobacter rodentium, suffered a lethal colitis coincident with severe defects in TJ-associated IEC integrity.

We next tested whether in vivo TLR2 stimulation exerts specific TJ-protective effects in the intestinal epithelium, which could be used to treat stress-induced barrier damage in murine colitis. ${ }^{12}$ After DSS termination on day 6 , we administered a TLR2 ligand orally in drinking water for 4 or 6 subsequent days. TLR2-treatment significantly ameliorated all clinical signs of DSS-induced colonic inflammation (including weight loss, rectal bleeding, and mortality), whereas DSS-control mice 
showed marked colitis-associated signs on day 12. The TLR2 ligand PCSK attenuated severity of DSS colitis as assessed by any of the several parameters including colon length and histopathology. Mechanistically, PCSK-mediated clinical amelioration correlated with significant TJ preservation and decrease of intestinal permeability. Intestinal epithelial ZO- 1 architecture was substantially preserved, even in close proximity to inflammatory infiltrates, and serum levels of fluorescein isothyocyanatedextran were significantly lower in those DSS mice treated with PCSK - comparable to healthy mice. In contrast, DSS-control mice without PCSK therapy showed significant loss of association and disruption of $\mathrm{ZO}-1$ from the lateral $\mathrm{TJ}$ membrane in regenerating IEC and increased serum levels of fluorescein isothyocyanate-dextran. No significant apoptosis was observed in regenerating IEC of either DSS-alone or PCSK-treated DSS mice. Collectively, these studies ${ }^{11,12}$ imply that functional TLR2 is necessary to preserve intestinal epithelial TJ-associated integrity against toxic or inflammatory stress-induced damage indicating that the host injury response requires stimulation by microbegenerated products to actively maintain epithelial barrier homeostasis in vivo.

However, it is unlikely that TLR2 stimulation opposes mucosal inflammation solely through its effects on IEC. TLR2 is functionally expressed by many cells within the intestinal mucosa (Figure 2). Injection of TLR2 ligands into the gut lumen has been shown to induce migration of subepithelial DCs and promote antigen capture by follicle-associated epithelium. ${ }^{38} \mathrm{As}$ outlined above,TLR2-mediated IL-10 production in LPMNC represents an additional key homeostatic mechanism that critically serves to limit proinflammatory cytokine production and tissue damage in the intestinal mucosa. In fact, it has recently been demonstrated that oral administration of LcrV-secreting Lactococcus lactis induces IL-10 via TLR2, thus preventing immune-mediated 2,4,6-trinitrobenzene sulfonic acid colitis, ${ }^{39}$ yet the major cellular target of TLR2 remains to be identified TLR2 also positively modulates regulatory $\mathrm{T}$ cells in the intestinal mucosa. TLR2-stimulated CD4 + CD25 + regulatory T cells have been shown to ameliorate spontaneous colitis induced by effector $\mathrm{T}$ cells in severe combined immunodeficient mice. ${ }^{40}$ Yet, the role of TLR2 signaling and its adaptor molecule MyD88 in distinct induction of $\mathrm{T}_{\mathrm{H}} 1 / \mathrm{T}_{\mathrm{H}} 2$ immune responses appears to be ambiguous. ${ }^{41-43}$ Taken together, one must acknowledge that the barrier-protective mechanisms via intestinal epithelial TLR2 may be supplemented by anti-inflammatory effects of distinct immune cell-cell crosstalks in the subjacent submucosa, which need to be further defined in future studies.

\section{CONCLUSION}

Our studies collectively provided first evidence of TLR2-mediated control of mucosal inflammation by regulating TJ-associated intestinal epithelial barrier integrity. TLR2 stimulation directly enhances IEC barrier function via certain PKC isoforms in vitro. TLR2 deficiency predisposes to stress-induced injury of TJ-modulated barrier function leading to perpetuation of mucosal inflammation and apoptosis. TLR2-induced TJ modulation strongly interrelates with promotion of IEC survival through the PI3K/Akt-pathway. Finally, these studies imply that TLR2 represents a potential therapeutic target for the treatment of gastrointestinal disorders, ${ }^{44}$ in which TJ-associated intestinal epithelial barrier disruption may represent a predominant pathogenetic feature.

\section{ACKNOWLEDGMENTS}

This study was supported by grants from the Crohn's and Colitis Foundation of America (SRA), the Deutsche Forschungsgemeinschaft (Ca226/4-2), and the IFORES bonus program (Medical Faculty of Essen).

\section{DISCLOSURE}

The author has declared no financial interests.

(c) 2008 Society for Mucosal Immunology

\section{REFERENCES}

1. Meddings, J. Barrier dysfunction and Crohn's disease. Ann. NY Acad. Sci. 915, 333-338 (2000)

2. Schmitz, H. et al. Altered tight junction structure contributes to the impaired epithelial barrier function in ulcerative colitis. Gastroenterology 116, 301-309 (1999).

3. Perara, L. \& Mayer, L. Immunologic defects underlying the pathogenesis of IBD. Gastroenterol. Hepatol. 1, 108-116 (2005).

4. Xavier, R.J. \& Podolsky, D.K. Unravelling the pathogenesis of inflammatory bowel disease. Nature 448, 427-434 (2007).

5. Boivin, M.A. et al. Mechanism of glucocorticoid regulation of the intestinal tight junction barrier. Am. J. Physiol. Gastrointest. Liver Physiol. 292, G590-G598 (2007)

6. Hooper, L.V. et al. Molecular analysis of commensal host-microbial relationships in the intestine. Science 291, 881-884 (2001).

7. Madsen, K. et al. Probiotic bacteria enhance murine and human intestinal epithelial barrier function. Gastroenterology 121, 580-591 (2001).

8. Otte, J.M., Cario, E. \& Podolsky, D.K. Mechanisms of cross hyporesponsiveness to Toll-like receptor bacterial ligands in intestinal epithelial cells. Gastroenterology 126, 1054-1070 (2004).

9. Cario, E. et al. Lipopolysaccharide activates distinct signaling pathways in intestinal epithelial cell lines expressing Toll-like receptors. J. Immunol. 164, 966-972 (2000).

10. Cario, E. \& Podolsky, D.K. Differential alteration in intestinal epithelial cell expression of toll-like receptor 3 (TLR3) and TLR4 in inflammatory bowel disease. Infect. Immun. 68, 7010-7017 (2000).

11. Cario, E., Gerken, G. \& Podolsky, D.K. Toll-like receptor 2 enhances ZO-1-associated intestinal epithelial barrier integrity via protein kinase C. Gastroenterology 127, 224-238 (2004).

12. Cario, E., Gerken, G. \& Podolsky, D.K. Toll-like receptor 2 controls mucosal inflammation by regulating epithelial barrier function. Gastroenterology 132, 1359-1374 (2007).

13. Cario, E. Bacterial interactions with cells of the intestinal mucosa: Toll-like receptors and NOD2. Gut 54, 1182-1193 (2005).

14. Janeway, C.A. Jr. \& Medzhitov, R. Innate immune recognition. Annu. Rev. Immunol. 20, 197-216 (2002).

15. Bessler, W.G. et al. Synthetic lipopeptide analogs of bacterial lipoprotein are potent polyclonal activators for murine B lymphocytes. J. Immunol. 135, 1900-1905 (1985).

16. Buwitt-Beckmann, U. et al. TLR1- and TLR6-independent recognition of bacterial lipopeptides. J. Biol. Chem. 281, 9049-9057 (2006).

17. Jin, M.S. et al. Crystal structure of the TLR1-TLR2 heterodimer induced by binding of a tri-acylated lipopeptide. Cell 130, 1071-1082 (2007).

18. Gantner, B.N., Simmons, R.M., Canavera, S.J., Akira, S. \& Underhill, D.M Collaborative induction of inflammatory responses by dectin-1 and Toll-like receptor 2. J. Exp. Med. 197, 1107-1117 (2003).

19. Hoebe, K. et al. CD36 is a sensor of diacylglycerides. Nature 433, 523-527 (2005).

20. Pierik, M. et al. Toll-like receptor-1, -2 , and -6 polymorphisms influence disease extension in inflammatory bowel diseases. Inflamm. Bowel Dis. 12, 1-8 (2006)

21. Arbibe, L. et al. Toll-like receptor 2-mediated NF-kappa B activation requires a Rac1-dependent pathway. Nat. Immunol. 1, 533-540 (2000). 
22. Yamamoto, M. et al. Essential role for TIRAP in activation of the signalling cascade shared by TLR2 and TLR4. Nature 420, 324-329 (2002).

23. Shibolet, O. et al. AKAP13 a rhoa GTPase-specific guanine exchange factor is a novel regulator of TLR2 signaling. J. Biol. Chem. 282, 35308-35317 (2007).

24. Shkoda, A. et al. Interleukin-10 blocked endoplasmic reticulum stress in intestinal epithelial cells: impact on chronic inflammation. Gastroenterology 132, 190-207 (2007).

25. Re, F. \& Strominger, J.L. IL-10 released by concomitant TLR2 stimulation blocks the induction of a subset of Th1 cytokines that are specifically induced by TLR4 or TLR3 in human dendritic cells. J. Immunol. 173, 7548-7555 (2004).

26. Yanagawa, Y. \& Onoe, K. Enhanced IL-10 production by TLR4- and TLR2-primed dendritic cells upon TLR restimulation. J. Immunol. 178, 6173-6180 (2007)

27. Zanin-Zhorov, A. et al. Heat shock protein 60 enhances CD4+ CD25+ regulatory $T$ cell function via innate TLR2 signaling. J. Clin. Invest. 116, 2022-2032 (2006).

28. Imanishi, T. et al. Cutting edge: TLR2 directly triggers Th1 effector functions. J. Immunol. 178, 6715-6719 (2007).

29. Watanabe, T., Kitani, A., Murray, P.J. \& Strober, W. NOD2 is a negative regulator of Toll-like receptor 2-mediated T helper type 1 responses. Nat. Immunol. 5, 800-808 (2004).

30. $\mathrm{Hu}, \mathrm{X}$. et al. IFN-gamma suppresses IL-10 production and synergizes with TLR2 by regulating GSK3 and CREB/AP-1 proteins. Immunity 24 , 563-574 (2006).

31. Melmed, G. et al. Human intestinal epithelial cells are broadly unresponsive to Toll-like receptor 2-dependent bacterial ligands: implications for host-microbial interactions in the gut. J. Immunol. 170, 1406-1415 (2003)

32. Boone, D.L. et al. The ubiquitin-modifying enzyme $\mathrm{A} 20$ is required for termination of Toll-like receptor responses. Nat. Immunol. 5, 1052-1060 (2004).
33. Mitic, L.L. \& Anderson, J.M. Molecular architecture of tight junctions. Annu. Rev. Physiol. 60, 121-142 (1998).

34. Itoh, M., Nagafuchi, A., Moroi, S. \& Tsukita, S. Involvement of ZO-1 in cadherin-based cell adhesion through its direct binding to alpha catenin and actin filaments. J. Cell. Biol. 138, 181-192 (1997).

35. Li, W. et al. Protein kinase C-alpha overexpression stimulates Akt activity and suppresses apoptosis induced by interleukin 3 withdrawal. Oncogene 18, 6564-6572 (1999).

36. Rakoff-Nahoum, S., Paglino, J., Eslami-Varzaneh, F., Edberg, S. \& Medzhitov, R. Recognition of commensal microflora by toll-like receptors is required for intestinal homeostasis. Cell 118, 229-241 (2004).

37. Gibson, D.L. et al. Toll-like receptor 2 plays a critical role in maintaining mucosal integrity during Citrobacter rodentium-induced colitis. Cell Microbiol. 10, 388-403 (2008)

38. Chabot, S.M. et al. TLR2 activation by proteosomes promotes uptake of particulate vaccines at mucosal surfaces. Vaccine 25, 5348-5358 (2007)

39. Foligne, B. et al. Prevention and treatment of colitis with Lactococcus lactis secreting the immunomodulatory Yersinia LcrV protein. Gastroenterology 133, 862-874 (2007).

40. Liu, H., Komai-Koma, M., Xu, D. \& Liew, F.Y. Toll-like receptor 2 signaling modulates the functions of CD4+ CD25+ regulatory T cells. Proc. Natl. Acad. Sci. USA 103, 7048-7053 (2006).

41. Redecke, V. et al. Cutting edge: activation of Toll-like receptor 2 induces a Th2 immune response and promotes experimental asthma. J. Immunol. 172, 2739-2743 (2004).

42. Patel, M. et al. TLR2 agonist ameliorates established allergic airway inflammation by promoting Th1 response and not via regulatory $T$ cells. J. Immunol. 174, 7558-7563 (2005)

43. Rakoff-Nahoum, S., Hao, L. \& Medzhitov, R. Role of Toll-like receptors in spontaneous commensal-dependent colitis. Immunity 25, 319-329 (2006).

44. Cario, E. Therapeutic impact of toll-like receptors on inflammatory bowe diseases: a multiple-edged sword. Inflamm. Bowel Dis. 14, 411-421 (2008). 